Research Article

\title{
Submarine Cable Detection Method Based on Multisensor Communication
}

\author{
Yikang Chen $\mathbb{D}^{1,2}$ Xiaojun Li, ${ }^{1,2}$ Chi Cai, ${ }^{1,2}$ Cong Wu, ${ }^{1,2}$ Weijia Zhang, ${ }^{1,2}$ Xiaowei Huang, ${ }^{1,2}$ \\ Qiang Guo, ${ }^{1,2}$ and Daoyu Jiang ${ }^{1,2}$
}

${ }^{1}$ Extra High Voltage Power Transmission Company, China Southern Power Grid Co., Ltd, Guangdong, Guangzhou 510000, China

${ }^{2} J o i n t$ Laboratory of DC Transmission Equipment and Submarine Cable Safety Operation, China

Correspondence should be addressed to Yikang Chen; cyk@hainanu.edu.cn

Received 30 September 2021; Accepted 30 November 2021; Published 21 December 2021

Academic Editor: Gengxin Sun

Copyright ( 2021 Yikang Chen et al. This is an open access article distributed under the Creative Commons Attribution License, which permits unrestricted use, distribution, and reproduction in any medium, provided the original work is properly cited.

\begin{abstract}
Submarine cable is widely used in today's oil industry, and it is a much hidden large-scale industrial facility, which vigorously promotes the development of people's lives. With the widespread use of submarine cables based on multisensor communication, as far as the current situation is concerned, this paper makes a report and summary on the research of submarine cable detection method in shallow sea area (sea area within $200 \mathrm{~m}$ ). According to the implementation of the project and the way of controlling variables, the current common detection modes are planned, fault prediction, fault diagnosis, fault analysis and summary, and experimental data comparison, and then, we can use Brillouin radio frequency to prevent the occurrence of submarine cable fault, and when the fault occurs, we can detect the fault at the first time. The feedback value range of TTSL electromagnetic detection is very stable, and the Brillouin scattering frequency is within the normal fluctuation range. In deep-sea exploration, TTSL electromagnetic detection can detect faults for submarine cables and will not affect the fault in all aspects of waveform and wavelength. Finally, the best path and future development trend of submarine cable detection method are obtained by analyzing and summarizing the detection data, and a complete scheme plan such as some preventive measures and repair technology is put forward.
\end{abstract}

\section{Introduction}

With the rapid development of science and technology, the development of national marine economy is also an important strategic goal to support national development. China has a vast sea area and abundant offshore resources, and a large number of submarine cables are needed for power transportation and communication between islands, inland and offshore platforms. Compared with the tunnel and support laying of land cables, most of the submarine cables are laid on the seabed and have the characteristics of large capacity and long distance. Therefore, the fault detection of submarine cable has become an important task of marine communication. At present, submarine cables for multisensor communication are distributed in many countries and regions; across many oceans, it has far-reaching influence and significance on contemporary social progress. It pro- motes the development of human world civilization and allows people to look forward to the future, which also saves most of the space and saves a lot of space resources for human activities in areas where human beings live [1]. However, the detection methods of submarine cables are emerging one after another at present. From the initial detection methods to today's echo signal detection, sonar detection, underwater photography and other methods, a lot of research has been done on the detection methods of submarine cable. Each method has its own advantages and disadvantages, and it is a good method to weigh the advantages and disadvantages through data research [2]. This is the further research and data analysis of submarine cable detection method for multisensor communication. Sensor fusion technology can greatly reduce the processing speed of data processing and can effectively avoid blind areas and misunderstandings of optical detection. This reduces the cost. 
The method is more effective. The detection speed is faster and more accurate [3]. At present, a very effective technology for submarine cable damage has been developed to the final stage. This technology is to manufacture an XLPE submarine large cable [4] and carry out various test functions such as compression test and then use optical fiber to transmit it to the test sensor to greatly improve the test quality [5]. The combination of cable and equipment is cable connection with outer skin, and outer skin is insulator, which has the function of high strength and pressure resistance, which makes the selection of materials particularly important, changes the method and research of detecting multisensors, and also has some influence on various data: temperature, pressure, impact volume, pressure test, etc. [6]. Thousands of submarine sensor communication cables have been crossing the world's oceans. Our unknown broadband, power generation, and so on are due to the effectiveness of these submarine cables, which provide us with energy supply along the $50 \mathrm{~km}$ relay station, which cannot be underestimated [7]. In order to detect the damage of submarine cables, it is suggested to put forward the principle of echo signal reflection. Ultrasonic waves are very fast, so when they touch submarine cables, they can resonate and send feedback as quickly as possible [8]. This detection method does not need to worry about obstacles in the middle and some troubles caused by bad weather [9]. In this way, the continuous detection of submarine multisensor communication cables under pressure and harsh environment is solved [10]. In order to study the maintenance work and technology of submarine cable, combined with the engineering situation, the detection and repair technology of submarine cable is caused, and reasonable suggestions are put forward according to the research of detection methods [11]. This is also the importance of multisensor communication submarine cable. The detection speed and quality are often inversely proportional, and it is difficult to achieve both. In this paper, when analyzing and constructing multisensor communication submarine cable, there are some fault detection, fault analysis, fault simulation, fault diagnosis, and other aspects of variable control to obtain various data. Through the analysis of data, this paper summarizes the understanding of submarine cable in the research report on submarine cable detection method [12]. Because submarine cable is a large and hidden facility, it is difficult for people to see it in daily life, and many people often have a wrong understanding and detection understanding of submarine cable [13]. So in order to correct people's misunderstanding, this paper corrects some people's misconceptions about submarine cables at present. It also enables people to better understand that the implementation of the multisensor communication submarine cable inspection can also make the submarine cable move forward stably in today's society and even in the future development [14]. Scientific development will make more profitable contributions to the future of mankind. Most sea areas have not yet been developed, which is also the bottleneck of submarine cables [15]. When the implementation and testing of submarine cables are more comprehensive and the project is larger, I believe that science and technology will make a greater leap. Due to the high equipment investment and engineering cost of submarine cables, when damage occurs, it will inevitably cause greater economic losses to the communication industry. Therefore, it can quickly detect submarine cable faults and detect abnormal conditions, propose solutions, and quickly remove faults, which has great guidance and reference significance for improving maintenance efficiency, reducing economic losses, and restoring production.

\section{Fault Detection}

Submarine cables usually have to pass through land, trestle bridge, shoal, and some particularly complex terrain seabed with short distance and length. However, different sections of the same submarine cable will have different Brillouin dispersion radio frequency shifts, and this difference value will produce uncontrolled fluctuations in a certain range. Therefore, a single threshold variable is completely unable to realize fault feedback of submarine cables. In this paper, the fault detection and feedback of multisensor communication submarine cable are completed through long-term monitoring, supervision, and setting of two or even more thresholds and multiple variables.

When the submarine cable did not fail, the Brillouin radiofrequency shift data of each stretching band of optical fiber in submarine cable for multisensor communication are relatively stable and difficult to fluctuate. However, according to the accumulated load of optical fiber for a long time, the temperature difference data in seawater, and the historical threshold detection waveform results, the standard wavelength of submarine cable is $\operatorname{Se}(U)$.

The alarm threshold of ship cable determines $Y K$ according to the actual situation and fault experience of ship cable. Taking the standard wavelength $\operatorname{Se}(U)$ as the central axis and as shown in Figure 1, $Y K$ as the normal fluctuation range, it can define its normal operation interval, and as long as it exceeds the normal operation range, it is the fault difference point.

When the number of discrepancy points detected twice or more times exceeds the normal data alarm threshold, the alarm will be sent out, the alarm time and data will be recorded, and fault diagnosis will be submitted.

Fault discrimination of connecting ground. In the event of a ground connection failure, the Brillouin radio frequency shift of the fiber between the ground point and the power supply will far exceed the dangerous threshold $g t_{2}$ of the ground connection. $N$ meter monitoring data $\mathrm{Smon}_{1}$ and standard data $\mathrm{SN}$ are taken from the power supply side to the end-user side. If $\operatorname{Smon}_{2}(X)-\mathrm{SN}(\mathrm{X})>g t h_{2}$, they are considered to be grounding singularities, where $X \in(1, N)$; when the number of singular grounding points NUM$G R O_{2}$ exceeds $0.95 \mathrm{~N}$, the grounding fault is determined; otherwise, the next step is determined.

For nongrounded fault signals, dune wave decomposition is carried out. In order to detect the mutation point most effectively and directly, the selected dune wave base must have a sufficiently high vanishing matrix when the immediate analysis produces the mutation signal. At this moment, the waveform and wavelength of Brillouin radio 


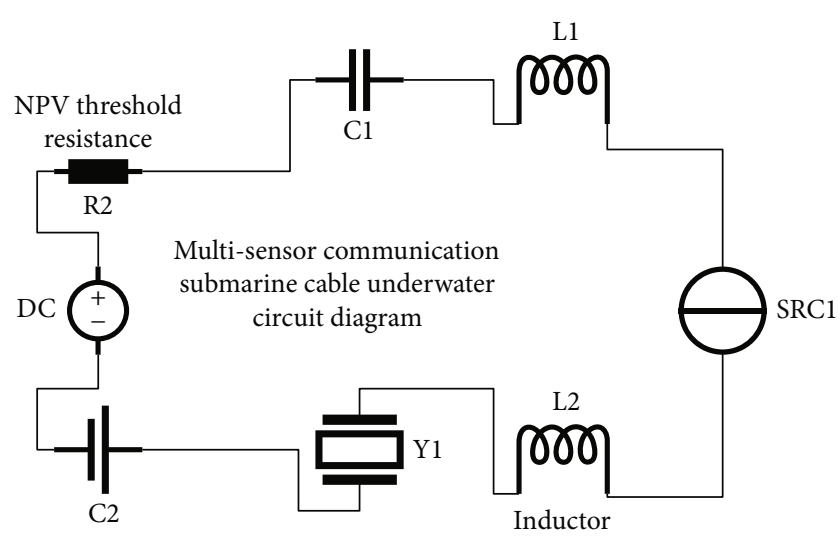

Figure 1: Circuit diagram of multisensor communication submarine cable underwater.

frequency shift in multisensor communication submarine cable are quite rough and unstable, determine the uneven dune wave pattern, that is, the low frequency wave with huge exploratory coefficient and low regularity, and use different dune wave bases to test and compare repeatedly. Finally, we can determine the ccoif_2 dune wave base section and decompose the alarm signal at multiple scales. In this way, it can diagnose faults in a superlarge range and can also be used for interval detection. It can have more range choices and higher fault tolerance rate, and the detection results achieved in this way are the most convincing.

Fault confirmation. Explore and find the model maximum fixed point of each scale, if one or more modulus maximum points with very large amplitude are found on the $J-1$ scale, and the model maximum points with the same sign domain can be found in the coordinate points adjacent to the abscissa scale position of $J-1$ on the vertical axis scale of $J$, this can explain and determine the same mutation outlier point corresponding to these two horizontal and vertical coordinate point domains, and on the same maximum line, if the next signal indicates that the maximum feature and maximum feature on $J-1$ scale are more prominent, then a judgment can be made, and the judgment content is to make it clear that there is a fault at the value point.

Fault diagnosis of overheating caused by anchor damage and partial discharge. The high-frequency coefficients of microwave decomposition are used to distinguish anchor damage faults from overheating faults caused by local power generation. Whenever the anchor fault occurs, the highfrequency coefficients $D 6$ and $D 7$ of the sixth and seventh scales will appear very obvious peak images at the occurrence position when the fault occurs, exceeding the alarm thresholds of high-frequency coefficients Wave_D5 and Wave_D7; as long as the overheating fault caused by partial discharge occurs, the high-frequency coefficients $D 4$ and D5 of the fourth and fifth scales will appear obvious peaks at the fault location, exceeding the alarm thresholds Wave_D4 and Wave_D5 of high-frequency coefficients.

False alarm elimination. After wavelet waveform and dune wave wavelength analysis, $D 4$, there will be no spike images in $D 5, D 6$, and $D 7$. There will be no maximum point, or when the alarm threshold number of high-frequency coefficient area is not reached, this shows that submarine cables are relatively safe. However, when error alarm occurs in the process of fault diagnosis, the system will modify the standard value interval according to the normal monitoring data in the latest week, so as to optimize the threshold interval of fault diagnosis and improve the accuracy and randomness of submarine cable fault monitoring.

\section{System Test Analysis}

3.1. Evaluation Index Model. Weight index of multisensor communication submarine cable in unstable seawater.

$$
(x+\beta)^{n}=\sum_{\alpha=0}^{n}\left(\begin{array}{l}
n \\
k
\end{array}\right) x^{6} a^{9.78-\beta},
$$

where $\beta$ represents the degree of the angle between the axis of the armor wire and the axis of the cable, and $a$ is an amount that varies approximately linearly with temperature within a certain range.

Brillouin dispersion radio frequency in

$$
\partial_{B}(v)=\partial 0 \frac{(\Delta \omega / 2)^{2}}{\left(v-v_{B}\right)^{2}+(\Delta \omega / 2)^{2}},
$$

where $v_{B}$ is Brillouin frequency shift and $\Delta \omega$ is Brillouin gain bandwidth.

The relationship between the pressure and the temperature of the submarine cable is shown in Figure 2.

In Formula (1), $n$ is the seabed pressure coefficient; when the pressure coefficient is constant, the weight of submarine cable can be calculated and the periodic frequency of sensing coefficient can be obtained. Brillouin is a series of inelastic light scattering caused by acoustic phonons in the medium. The frequency $V_{1}$ of scattered light relative to incident light is determined by the elasticity of acoustic characteristics. In addition, incident light $V_{1}$ is related to scattering angle $\alpha$.

$$
V a=V_{0}-V_{1}=\frac{2 V_{0}\left(n / \lambda_{0}\right)}{\operatorname{Sin}(\theta / 2)}
$$

where $V_{0}$ is the frequency of scattered light, $n$ is the refractive index of the medium, $\lambda_{0}$ is the wavelength of the incident light, and $\theta$ is the angle between the incident light and the Stokes light, and Brillouin frequency shift $v_{B}$ is determined by the following:

$$
v_{B}=\frac{2 n}{\lambda_{0}} \sqrt{\frac{(1-k) E}{(1+k)(1-2 k) p}},
$$

where $E$ and $K$ are Young's model, Poisson's ratio, and density of medium, respectively. For quartz fiber medium, because the frequency of front scattering light is 0 , only the frequency of backscattering light is considered.

(a) Brillouin scattering periodic frequency 


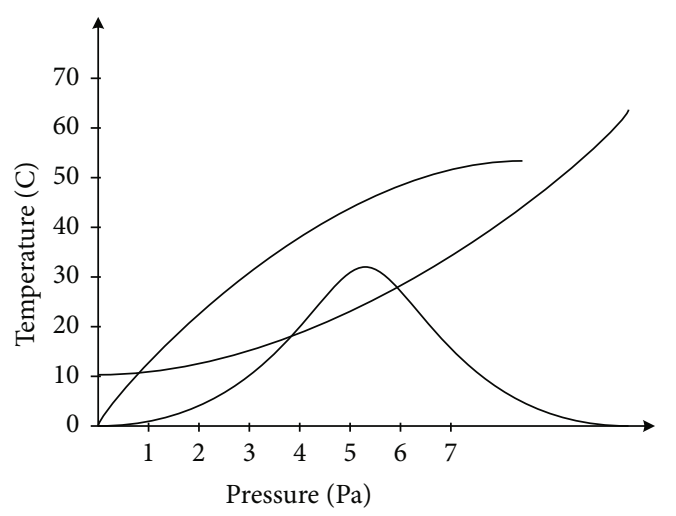

FIGURE 2: Brillouin scattering curve.

If the light is incident on the core-envelope interface at any angle $\varphi\left(\varphi c_{1}<\varphi<\pi / 2\right)$, select the point A1 on the core-envelope interface and any scattering point B1 for analysis, as shown in the figure. At A1, there is $\pi / 2+\varphi \leq \theta m n_{1}$ $\leq \pi$; at point $\mathrm{B} 1$, there is $\varphi c_{3}+\varphi \leq \theta m n_{2} \leq \pi$. Because $\varphi c_{1}$ $<\varphi<\pi / 2, \varphi c_{3}+\varphi<\pi / 2+\varphi$, there is $\varphi c_{2}+\varphi \leq \theta m n_{2} \leq \pi$. From the above analysis, it can be seen that the maximum value range of Brillouin scattering angle is $2 \varphi c \leq \theta m n_{2} \leq \pi$ when the light is incident at the critical angle of total reflection, that is, the maximum value range of Brillouin scattering angle is $2 \varphi \mathrm{c} \leq \theta \mathrm{mn}_{3} \leq \pi$ :

$$
\begin{gathered}
\alpha=\sqrt{\partial^{2}-\theta} \leq \lim _{\theta \rightarrow \infty} \frac{\partial !}{\theta !(\theta-4.7) !}, \\
p_{1}(\partial)=\frac{M}{\pi R_{0} l_{0}(1-k \partial)^{2}(1+\partial)},
\end{gathered}
$$

where $\theta$ is the angle between the sensor and the cable axis.

The weight of armored submarine cable in the sea can be expressed by the formula, namely,

$$
W s=W-\left(\frac{\pi d_{2}}{4}\right)\left(\frac{1.025 t}{M_{3}}\right) .
$$

The weight of armored submarine cable in the sea can be expressed by the formula, namely,

$$
W s=W-\left(V_{1}+V_{2}+0.5 V_{3}\right)\left(\frac{1.025 t}{M_{3}}\right),
$$

where $W$ is the weight of the submarine cable in the air, $W_{s}$ is the weight of the submarine cable in the water, $V_{1}$ is the volume of the cable core per kilometer, and $V_{2}$ is the volume of the armored steel wire per kilometer.

\section{(b) Multisensor communication fusion computing}

The density and pressure exponent of submarine cable can be obtained by the sensor fusion calculation algorithm, and then, the failure exponent rate and detection rate of multisensor communication submarine cable can be obtained by Brillouin radio frequency calculation. The detection method is used to detect submarine cable.

$$
\begin{gathered}
\theta=-\iint \frac{\partial B}{\partial t} \overrightarrow{d s}, \\
\partial=\int(\overrightarrow{v \times \beta}) \cdot d \vec{l}, \\
\rho=\frac{d \varphi}{d t}=-\frac{d}{d t}\left[\iint \overrightarrow{B \cdot d s}\right] .
\end{gathered}
$$

Calculation model exponential rate of sensing detection frequency

$$
\begin{gathered}
\Delta_{\varepsilon}=\frac{k^{2}(0)[2-k(0)]}{[1-k(0)][1-2 x(0)]}\left(\Delta_{\varepsilon-1}\right), \\
E(\delta)=\frac{1}{r_{0}}\left(\frac{d^{2} u(r)}{d r^{2}}\right),
\end{gathered}
$$

where $d$ is the cable diameter, $r$ is the radius, and $B$ is the modulus of elasticity.

It can be seen that the required value can be obtained only by asking for it, and the sensing rate data can be obtained. Because $K$ is a constant, the Brillouin dispersion RF wavelength data of submarine optical cable can be obtained under the condition of constant scattered light frequency.

Calculate the resistance of submarine cable in the sea, get the pressure coefficient ratio, and then carry out further detection and fault prediction on submarine cable, which can accurately calculate the pressure interval and the stress area interval, namely,

$$
\begin{gathered}
U(r)=A\left(-\frac{1}{r^{2}}+e^{-k}\right), \\
p(\varepsilon)=\frac{M}{\pi R_{0} l_{0}}\left[\partial \frac{1}{\sqrt{\pi^{2}+\theta^{2}}}\right],
\end{gathered}
$$

where $U(r)$ is each group of atoms in Shi Ying optical fiber submarine cable, $R_{0}$ is a tetrahedron and a group, and the interaction between adjacent atoms is $r$; the data can be analyzed.

3.2. Fault Simulation. Due to the high price and special working environment, the experimental conditions of the entity are demanding, the research and development cycle is long, the cost is high, and the practical operation is difficult. In this paper, the characteristics of temperature difference, pressure, and strain capacity are obtained according to the long-term accumulated experience of submarine cable fault engineering measurement and analysis, and then, the normal detection data are corrected to simulate and restore the submarine fault scenario.

In the event of a failure of the submarine cable connecting the ground fault, the temperature of the workload fiber in the cable connecting the ground point to the seabed of the user's outer gap will translate linearly upward. The 


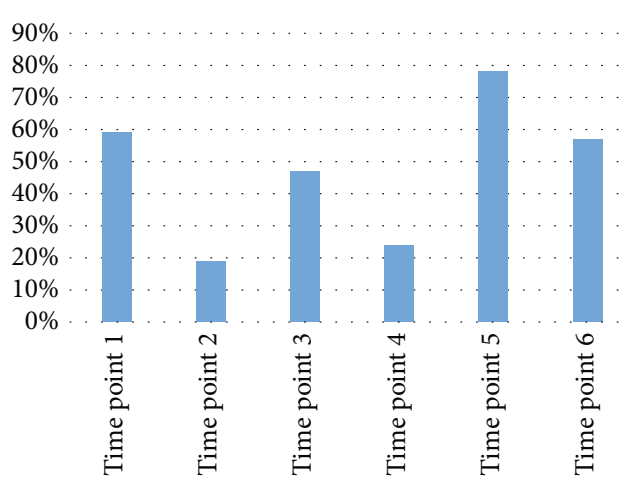

FIGURE 3: Histogram of scattering frequency detection experiment.

landing point of the connection ground is defined at the distance of $1925 \mathrm{~m}$ from the optical fiber, and grounding occurs from time 2. The maximum temperature rise of the optical fiber is $12^{\circ} \mathrm{C}$, and the temperature rise interval is $3^{\circ} \mathrm{C}$. The RF shift waveform of Brillouin dispersion is shown in Figure 3 . Time 1 is the frequency shift waveform at normal time, and time 2 to time 6 are the frequency shift flowing waveform after the connection ground fault occurs.

Due to the anchor failure, the submarine cable is damaged, and the optical fiber deformation value near the anchor point rises parabolically from the opening down. The optical fiber deformation value between the fixed point and the optical fiber 956 meters and 921 meters to 1040 meters is the $Y$ value within 50 meters and the normal change value of $X=\mathrm{AT}^{2}+B$. As the sum of the type values, the deformation values of other components are the normal deformation values of submarine cables, and the maximum modulation allowable capacity of submarine cable optical fibers is $2.7 \%$, which is equivalent to the frequency modulation of $0.64 \mathrm{GHz}$. As shown in Figure 4, the waveform with the length of Bria Mountain frequency modulation section of $0.05 \%$ is set. Two is the waveform of normal time, and from time 5 to time 9 is the waveform of the first five moments of the anchoring process.

Whenever a small part of overheated parts are damaged and anchored due to power generation, according to the experience of detecting cable sensing faults, the submarine cable at the unloading point is slightly worn locally, thus generating abnormally high heat. Once the temperature rises, the temperature on both sides will inevitably become higher. The point will gradually decrease with a little increase in distance, and the length of the temperature interval where large-scale anomalies occur will reach nanometer level. The parabola with downward opening can be used to express the temperature distribution area near the fault interval point. The distance between the entrance and exit points of the sensing communication submarine cable and the optical fiber can be defined as $2659 \mathrm{~m}$, and the temperature of the optical fiber interval between 1235 and $1679 \mathrm{~m}$ can be marked by the formula $X=-\mathrm{AT}^{2}+B$ and the sum of the normal temperature values between $1327 \mathrm{~m}$ and $X$. The temperature of other parts is the marine optical cable with normal temperature value, and the maximum tempera-

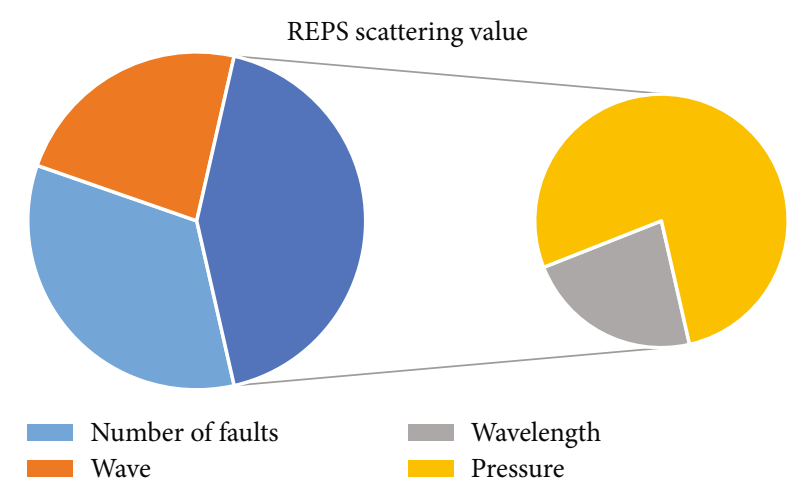

FIGURE 4: Fan-shaped experimental plot of REPS scattering values.

ture rise of the optical fiber at $X$ and $Y$ point is kept at 6.5331; Brillouin dispersive IF flowing waves and dune wavelengths are deformed as shown in Figure 3. In the figure, time point 2 is the normal time channel displacement waveform, and time $3 \sim$ time 6 is the prism waveform that generates heat after local small-scale discharge.

The overheating fault caused by bolt damage and partial discharge is superimposed on the overheating fault caused by bolt damage and partial discharge, forming a combined fault. The frequency shift waveform of Brillouin color scattering is shown in Figure 5, in which time 1 is the normal waveform, and time $2-6$ is the intermediate frequency shift waveform of anchoring and partial discharge simultaneously.

The main purpose of multibeam measurement is to analyze the main reasons for the exposure and suspension of offshore cables. Through multibeam timing measurement and auxiliary analysis of seabed sediment and complex terrain in submarine cable section, large-scale seabed sand slope in submarine cable line can be obtained. The height of sand slope is generally between 4 and $8 \mathrm{~m}$, and the wavelength is generally between $300 \mathrm{~m}$ and $700 \mathrm{~m}$ (the maximum wavelength is $700 \mathrm{~m}$ ). The flow surface has a long and gentle slope (generally between $3 \%$ and $7 \%$ ), while the return surface has a steeper slope (generally between $20 \%$ and $24 \%$ ). Most sand slopes have obvious asymmetry and nonlinearity, and twin sand waves are found on some large-scale sand waves. According to that analysis of the result obtained from the experiment, adverse geological phenomena of routing waves include sand, sand slope, scour trough, scour ridge and mound uplift, steep slope, landslide, coral reef, shallow buried rock, and weak stratum. In addition, the topography of submarine cable routing area is undulating and steep, with a large scale of gully bottom, convex mountain and bank slope, scouring pit, and sand wave. The cross-section topography is in the form of 90 Spend South Energy Construction Volume 4, with sand wave appearing in the whole line, and the ridgeline is generally north-south, perpendicular to Qiongzhou Strait tidal current (N-E). The shape, size, length, and wave height gradually increase from north to south. Therefore, the seabed sand slope and extremely complex flow pattern are the main reasons for the suspension of bare cables and the difficulty in fixing riprap. 


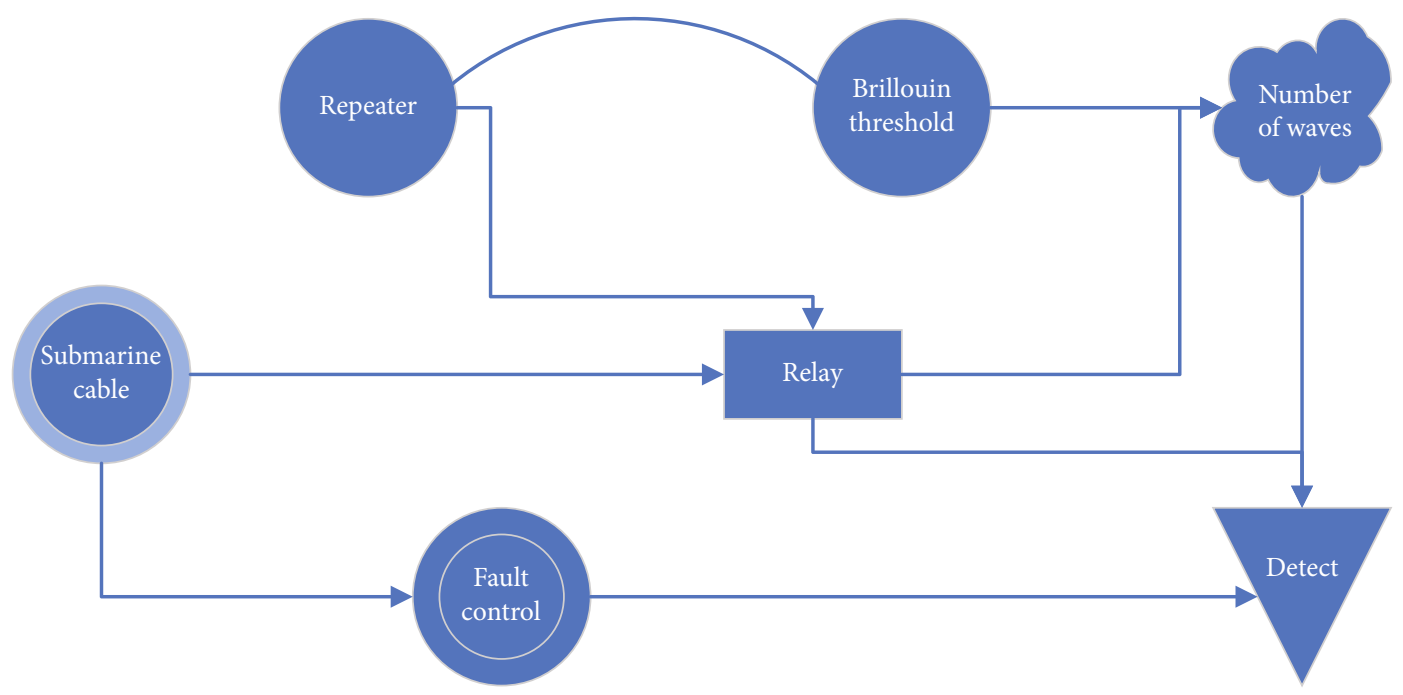

Figure 5: Flow chart of physical inspection of submarine cable.

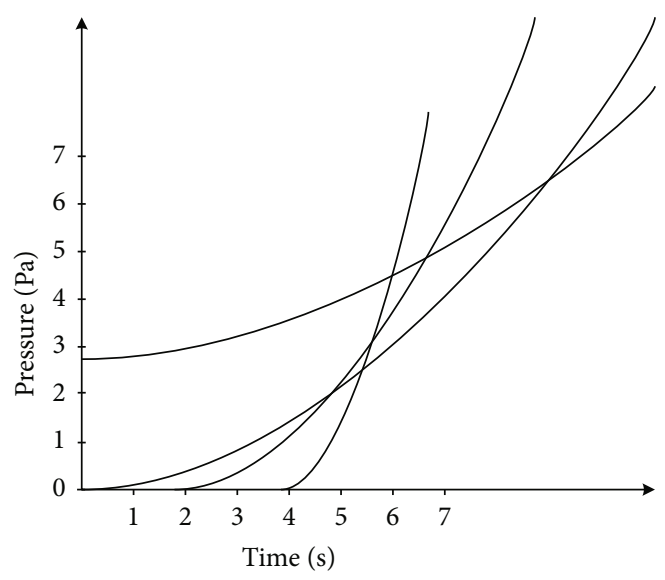

FIgURE 6: Function graph of sand dune wave on the axis of abscissa and ordinate.

3.3. Troubleshooting. According to the number of submarine cables $g t h_{2}=N F V=0.025$, the multisensor submarine cables are overloaded and overloaded. Finally, $\mathrm{Cgv}_{2}<\mathrm{IHP}$ $<g t h_{2}$ value is used to calculate the difference between small wavelength and large wavelength on the sand slope by using wave formula $P / R * 4.6>=N F V$. From the analysis of difference value and difference point, it is found that the submarine cables do not fail and the sensing system does not fluctuate abnormally.

Randomly choosing $\operatorname{coigh}_{2}=\mathrm{gth}_{2}$ value makes the decomposition theory of small wavelength of sand slope occur, which leads to the decomposition of wavelet into tiny particles.

Sand slope small wavelength analysis also shows that in the time scale from scale line 3 to scale line 5 in Figure 6, the value also changes and fluctuates greatly, resulting in abnormal power generation of the submarine cable and fluctuation caused by the decrease of the sensing degree of multisensor communication, indicating that the submarine cable fails in this time period, resulting in fault feedback.
The threshold wavelength of the alarm point for setting the high-frequency coefficient is $D 4=0.004 \mathrm{GHz}$, waveform $D 5=0.006 \mathrm{GHz}$, waveform $D 6=0.003 \mathrm{GHz}$, waveform $D 7$ $=0.005 \mathrm{GHz}$, and wave $D 7=0.005 \mathrm{GHz}$. The highfrequency coefficients of the 4 th and 5 th order are D3 $>D 4$ wave and $D 4>D 5$ wave near $1789 \mathrm{~m}$. In the vicinity of $1789 \mathrm{~m}$, the high-frequency coefficients of the 7th and 9th segments are D6 $>D 7$ and D4 $>D 5$ wave $D 5=0.003 \mathrm{GHz}$. Multisensor communication submarine cable can be completely discriminated as the anchor damage and overheating effect caused by local unified power generation. From Figure 6, it can be seen that the peak value of anchored dune fault on the superlarge spatial scale is the most prominent in the microwave fine decomposition ratios 5 and 8 . In wavelet decomposition ratios 6 and 7, there is a specific small part of discharge overheating, which leads to the dune fault having a particularly obvious peak value in a small space. Bolt breakage and local unloading are all completed according to the above steps. The results show that this method can effectively detect and diagnose the faults of multisensor communication submarine cables.

\section{Experimental Comparison}

4.1. Comparison of Mechanical Experiments. The pressure performance and environmental performance are tested, and the results are shown in Table 1.

The related test results are shown in Figures 7 and 8.

The pressure test is relatively stable and normal; control invariants and control variables will not cause wavelength waveform amplitude.

When the multisensor communication submarine cable is made of frequency conversion optical fiber, the detection methods are sonar detection, TTSL electromagnetic detection, side electromagnetic detection, ultrasonic detection, and deep-sea photography detection. The comparison of experimental results is shown in Figure 9.

The pressure of multisensor communication submarine cable in the detection method of submarine photography, 
TABLe 1: Pressure performance and environmental performance test.

\begin{tabular}{lcc}
\hline Object & Pressure performance & Environmental performance \\
\hline $\begin{array}{l}\text { GJB } \\
5589-\end{array}$ & $\begin{array}{c}\text { Overload working tension is normal; transient load index is normal; impact is } \\
122\end{array}$ & $\begin{array}{c}\text { The water seepage property is normal; the } \\
\text { temperature cycle is slow; the pressure index is } \\
\text { normal. }\end{array}$ \\
$\begin{array}{l}\text { GB/T } \\
18480\end{array}$ & $\begin{array}{c}\text { Overload working is normal; the transient load index is normal; the impact is } \\
\text { normal; flattening and repeated bending are normal. }\end{array}$ & $\begin{array}{c}\text { The watertight properties are normal; the } \\
\text { temperature cycle is fast; the pressure index is } \\
\text { overloaded. }\end{array}$ \\
\hline
\end{tabular}

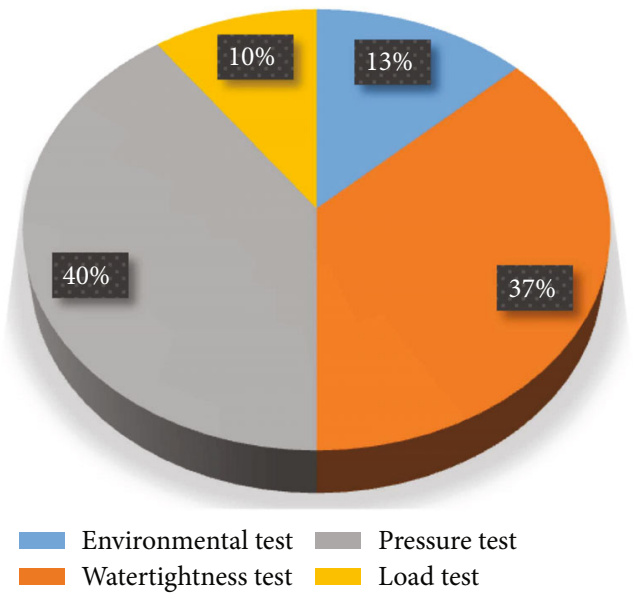

FIGURE 7: GJB mechanical experiment comparison data graph.

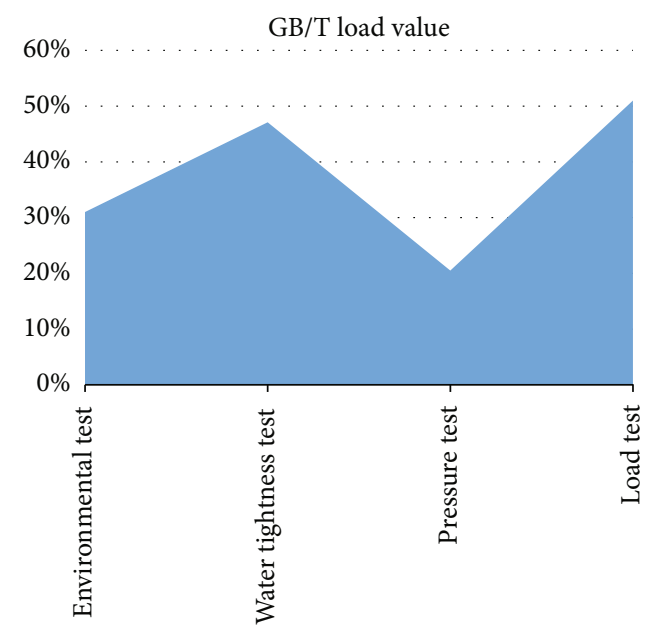

Figure 8: GB/T mechanical experiment comparison chart.

with the detection time, and fault feedback, the fault detection rate tends to be relatively stable, and waveform wavelengths tend to normal interval thresholds relatively, but the efficiency is very low. Compared with sonar detection technology and electromagnetic detection technology, it takes the longest time from the beginning to the end of detection, which leads to a limited number of detections. Moreover, when encountering complex seabed terrain and sand dunes and slopes, the seabed camera detection method is slightly weak, which greatly increases the detection difficulty and increases the detection risk in direct proportion. Fault damage is also obvious. When the number of faults is huge, the detection method of submarine camera cannot

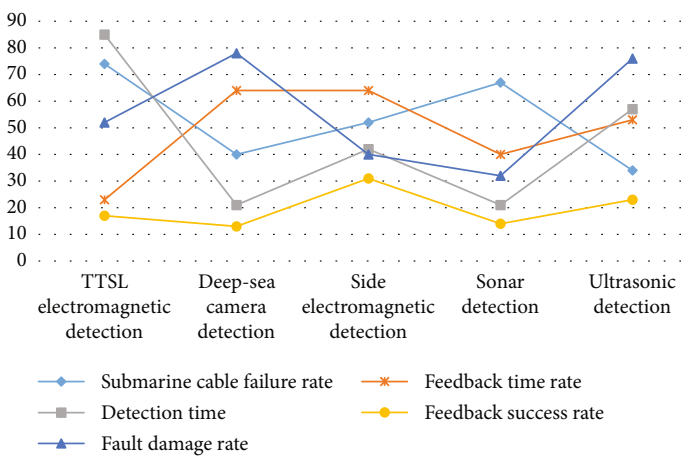

FIgURE 9: Comparative performance test of the five detection methods.

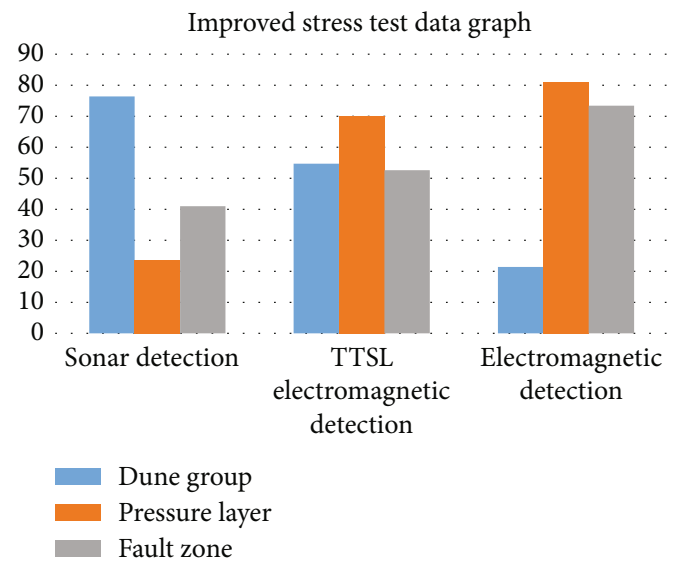

FIGURE 10: Improved stress test chart.

be used. Because the submarine pressure is too high in shallow sea, the submarine camera technology has no obvious abnormality, and the submarine camera sometimes cannot operate normally in the deep-sea test. The detection activity is temporarily terminated, which will make the experimental results produce errors, and the error range increases significantly in the range of $P \alpha * Z \beta$, so only the numerical interval error can be taken in Figure 10.

4.2. Pressure Contrast Test. For the pressure detection experiment of multisensor communication submarine cable on the seabed, in order to study whether the submarine cable has excessive pressure or pressure load exceeding normal value on the seabed, by studying the surface, submarine cables are in shallow sea areas. There is no difference in pressure values. All indexes of pressure are normal values. Waveform wavelength is also relatively normal. When 


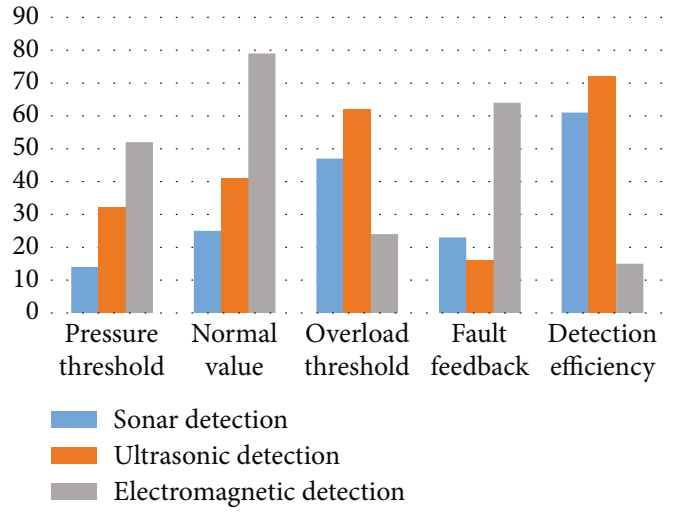

Figure 11: Three types of test data charts for submarine cables.

encountering complex seabed topography, there will be different choices and changes in the laying routes of submarine cables. The pressure threshold of submarine cable is normal at sand dunes, in a relatively stable state, as for that situation that there is no fluctuation in various data and the overload value increases, in order to evaluate the appropriate detection method, control relative variable method. Through the known sensing fusion formula, the scattering distribution map of Brillouin dispersion radio frequency is obtained. It can be seen from Figure 11 that the detection method can measure the pressure distribution of multisensor communication submarine cable on the seabed, which is convenient to measure and summarize the advantages and disadvantages of the detection method. The wavelet distribution, wavelength shape, and wavelength measurement are studied from the normal initial value, and the relevant conclusions are given for analysis and research. In order to further reduce the error, sonar detection technology, electromagnetic detection technology, and ultrasonic detection technology are used for experimental comparison, and the final comparison results are marked by the bottom line chart.

From the research data, it can be seen that for submarine cables with multisensor communication, sonar detection technology is not suitable for known submarine cables; for the damage of submarine cables, there is a positive advance. In view of this phenomenon, ultrasonic detection has performed abnormally well. For all aspects, wavelength fluctuation is relatively stable, and wavelet decomposition is relatively suitable and normal interval value in the region, which makes ultrasonic detection technology more comprehensive and rigorous.

4.3. Failure Rate of Short Load Detection. What is very common when working under short load in submarine cables, when large-scale demands such as large-scale power generation are needed, submarine cables will make short load working hours. At this time, when submarine cables are most prone to external interference and failure, usually, the radio frequency of Brillouin dispersion greatly exceeds the dangerous value. The submarine cable triggers emergency response measures. When the emergency response measures cannot relieve the power supply pressure, the submarine cable will generate large load power data, resulting in serious

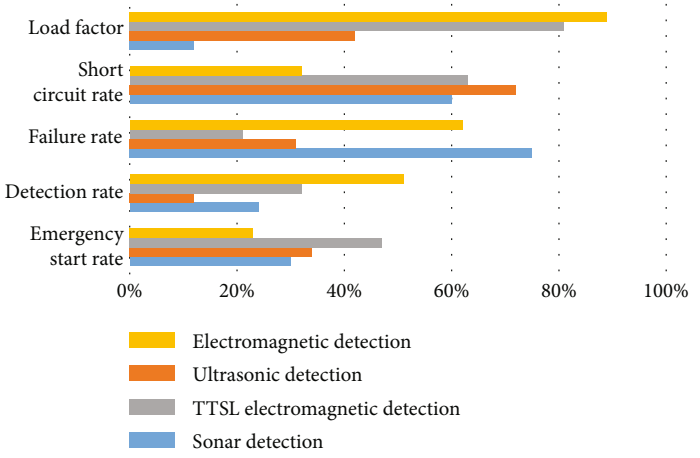

Figure 12: Short-term load detection diagram of multisensor submarine cable.

changes in wavelength waveform, which will pollute the submarine ecological environment, destroy the submarine ecological chain, and seriously cause irreversible anchor damage and large-scale short circuit. Therefore, short-term load detection of submarine cable failure rate becomes an important index, This is one of the major detection indexes of submarine cables, so for ultrasonic detection, sonar detection, electromagnetic detection, and TTSL electromagnetic detection are compared experimentally. The experimental process flow is the same as above. The control variable and invariant are short-term load rate, which increases the sensing rate of submarine cable and the working index and workload of submarine cable to achieve the effect of onehour short-term load and starts detection. The statistical comparison detection results are shown in Figure 12.

It is concluded that when the load rate is constant, the detection rate is roughly the same. However, sonar detection technology shows drawbacks, a sharp decline. There are also many shortcomings in the display of waveform wavelength. It is not suitable for testing under short load. Other detection methods, such as the battery detection method, TTSL electromagnetic detection method, and ultrasonic detection method, will be relatively balanced, and the Brillouin dispersion wavelength rate will not change obviously. Therefore, these three detection methods can be used to detect multisensor communication submarine cables in shallow sea areas. In the submarine cable testing experiment, the data can be obtained by using the above evaluation model formula, and the data can be converted into percentages to make the experimental results more significantly reflect the advantages and disadvantages of each testing method.

4.4. Evaluation Results. To sum up, in the multisensor communication submarine cable detection method, Brillouin value judgment is used to grade the feedback rate of detection; the three elements of feedback value and feedback period are attributed to TTSL electromagnetic detection technology commonly used at present. Electromagnetic detection, sonar detection, ultrasonic detection, and submarine camera detection adopt rigorous detection methods such as controlling the number of faults, controlling the depth of shallow sea and controlling variables. Finally, the evaluation results of each detection method are obtained, 
because the depth of shallow sea is similar. For seawater, density requirements tend to normal level. Sonar detection and submarine camera detection technology have good feedback values for submarine cable detection of multisensor communication in shallow sea field. Brillouin dispersion frequency fluctuation also tends to normal range, so it can be concluded that sonar detection and submarine camera are a good choice in shallow sea detection. For deep-sea detection, the feedback value range of TTSL electromagnetic detection is very stable, and Brillouin dispersion radio frequency is within normal fluctuation range. Therefore, TTSL electromagnetic detection can detect faults for submarine cables in deep-sea exploration, and the waveform and wavelength in all aspects will not affect the faults.

\section{Conclusion}

Because there are many ways to detect submarine cables in multisensor communication, but to sum up, this paper only uses several common methods to carry out fault feedback of submarine blue line detection. The final expected conclusion is obtained by data feedback, and the problems and defect directions of submarine cables are roughly predicted by fault prediction, so that the experiment can draw conclusions in a multivariable way, and a single variable cannot be used. The conclusions and results obtained by various variables are more convincing, scientific, and rigorous. This is also the charm of science. In this society with rapid development of science, a world with highly progressive humanities, I believe that the submarine cable for multisensor communication will be upgraded in higher technology in the future. Materials will be adopted more advanced, which also indicates that there will be more and more research on submarine cable detection in the future, and the detection types, detection methods, detection approaches, data flow analysis, and other methods will be more forward-looking and convincing, which is also the gospel of mankind and can make civilized by going up one flight of stairs. In this mysterious underwater world, I believe that there are not only the development prospects of sensing communication cables but also more opportunities and challenges, which is a necessary way for mankind to explore the world and science in the future. In the commercial market, submarine cable also has a place, and it exists in this market at a high price. Here, I also hope that the later detection methods will be studied with more and more rigorous scientific attitude.

\section{Data Availability}

The experimental data used to support the findings of this study are available from the corresponding author upon request.

\section{Conflicts of Interest}

The author declared that they have no conflicts of interest regarding this work.

\section{Acknowledgments}

This research was supported by the Science and Technology Project of China Southern Power Grid Co., Ltd. (CGYKJXM20180373).

\section{References}

[1] B. Smith, M. Hall, A. Franklin, E. S. Johansen, and O. Haldun Unalmis, "Field-wide installation of optical sensing technology yields multi-sensing benefits [J]," World Oil Magazine, vol. 229, no. 5, pp. 39-45, 2008.

[2] D. C. Shotton, "The radiography of polythene in submarine cable systems [J]," Proceedings of the IEE-Part A: Power Engineering, vol. 109, no. 3, pp. 199-202, 2010.

[3] Y. Li, L. Zhao, Z. Yang, A. Lv, and F. Wu, "Design and realization of the submarine cable three-dimensional monitoring system based on BOTDR [J]," Yi Qi Yi Biao Xue Bao/Chinese Journal of Scientific Instrument, vol. 35, no. 5, pp. 1029-1036, 2014.

[4] E. Ekici, Y. Gu, and D. Bozdag, "Mobility-based communication in wireless sensor networks," IEEE Communications Magazine, vol. 44, no. 7, pp. 56-62, 2006.

[5] C. Pandana and K. J. R. Liu, "Near-optimal reinforcement learning framework for energy-aware sensor communications," IEEE Journal on Selected Areas in Communications, vol. 23, no. 4, pp. 788-797, 2005.

[6] A. Sahari, T. T. Ruckh, R. Hutchings, and H. A. Clark, "Development of an optical nanosensor incorporating a $\mathrm{pH}$-sensitive quencher dye for potassium imaging," Analytical Chemistry, vol. 87, no. 21, pp. 10684-10687, 2015.

[7] M. Weyn, G. Ergeerts, L. Wante, C. Vercauteren, and P. Hellinckx, "Survey of the DASH7 Alliance Protocol for $433 \mathrm{MHz}$ wireless sensor communication," International Journal of Distributed Sensor Networks, vol. 9, no. 12, 2013.

[8] G. Tadesse, "Distributed particle filter for target tracking: with reduced sensor communications," Sensors (Basel), vol. 16, no. 9, p. 1454, 2016.

[9] J. M. Chen, R. Z. Lin, and Y. X. Sun, "On communication architecture for wireless sensor networks [J]," Chinese Journal of Sensors and Actuators, vol. 17, no. 3, pp. 197-208, 2006.

[10] R. Rahman, M. Alanyali, and V. Saligrama, "Distributed tracking in multihop sensor networks with communication delays," IEEE Transactions on Signal Processing, vol. 55, no. 9, pp. 4656-4668, 2007.

[11] T. Kasetkasem and P. K. Varshney, "Communication structure planning for multisensor detection systems," IEE Proc, Radar Sonar Navig, vol. 148, no. 1, pp. 2-8, 2001.

[12] W. L. Kruer, "Nonlinear estimates of Brillouin scatter in plasma," Physics of Fluids, vol. 23, no. 6, pp. 1273-1275, 1980.

[13] D. W. Phillion, W. L. Kruer, and V. C. Rupert, "Brillouin scatter in laser-produced plasmas," Physical Review Letters, vol. 39, no. 24, pp. 1529-1533, 1977.

[14] I. V. Bogachkov, "Research properties of the MandelstamBrillouin scatter in the polarization maintaining single-mode fibers [J]," Journal of Physics: Conference Series, vol. 1210, no. 1, article 012024, p. 7, 2019.

[15] N. T. Otterstrom, S. Gertler, Y. Zhou et al., "Backscatterimmune injection-locked Brillouin laser in silicon," Physical Review Applied, vol. 14, no. 4, 2020. 